\title{
A importância de um programa ludomotor e da estimulação cortical no desenvolvimento cinestésico de crianças
}

\author{
Alisson Padilha de Limaํㅜ Fabrício Bruno Cardoso²
}

\begin{abstract}
RESUMO
O estudo teve por objetivo avaliar o valor de um programa ludomotor combinado com a estimulação cortical voltado para o desenvolvimento cinestésico corporal de crianças. A pesquisa caracterizou-se por um estudo de campo experimental. O grupo participante foi composto por 150 crianças que foram divididas em três grupos. Como instrumento avaliativo utilizou-se Protocolo de avaliação de Bruininks-Oseretsky. Foi empregada uma análise de comparações múltiplas que demonstrou significância num sentido de comparação Pré X Pós teste. Conclui-se que a utilização combinada da estimulação cortical com um programa ludomotor favoreceu o desenvolvimento cinestésico corporal de crianças.
\end{abstract}

Descritores: Atividade motora, Crianças, Estimulação Auditiva.

\section{The importance of a ludomotor program and cortical stimulation in the development kinestésic of children}

\begin{abstract}
The study has the objective of evaluating the value of a ludomotor program combined with the cortical stimulation directed towards the kinestesic corporal development of children. The research focused on experimental field study. The participant group was composed by 150 children that were divided into three groups. As assessment tool was used Protocol Assessment Bruininks-Oseretsky. An analysis was used for multiple comparisons that showed significance in the sense of comparing X Pre Post Test. It is concluded that the combined use of cortical stimulation with a program ludomotor favored the development of children kinesthetic body.
\end{abstract}

Descriptors: Motor Activity, Children, Auditory Stimulation.

${ }^{1}$ Mestrando em envelhecimento humano pela Universidade do Passo Fundo (UPF), Passo Fundo, RS, Brasil

${ }^{2}$ Doutorando em ciências biológicas pela Universidade Federal do Rio de Janeiro (UFRJ), Rio de Janeiro, RJ, Brasil 


\section{Introdução}

Atualmente poucas pesquisas no âmbito da Educação Física vêm relacionando e identificando o valor de intervenções através da estimulação cortical e programas ludomotores que são caracterizadas pela atividade lúdica combinada com 0 ato motor, como melhora do repertório motor dessas crianças, onde 0 ato de brincar estimulado juntamente com 0 ato motor pode proporcionar condições adequadas ao seu desenvolvimento físico, motor e cognitivo ${ }^{1,2}$.

Através da combinação de duas intervenções essas crianças podem obter melhoras no seu desenvolvimento cinestésico corporal, que é entendido como o ato de controlar seu próprio corpo e manusear objetos com habilidade, potencializando e explorando a capacidade desses indivíduos em realizar uma tarefa motora que exija um maior grau de destreza ${ }^{3}$.

Pesquisas indicam que crianças estimuladas, de forma contextualizada, através de programas ludomotores, podem produzir redes de neurônios "exercitados"; e também um número maior de ramificações (dendritos) que se comunica com dendritos de outros neurônios, proporcionando, assim, que as informações proprioceptivas se consolidem na melhor maneira possível em seu córtex cerebral, proporcionando uma melhor integração neurofisiológica ${ }^{4,5,6}$.

A estimulação ludomotora faz com que a criança tenha como resultado uma memória motora, também conhecida como memória procedural ou de procedimentos, da qual depende, fundamentalmente, o aumento do desempenho que incrementa a precisão do gesto motor ${ }^{7,8,9,10}$.

Estudos nessa linha de aprendizagem apontam, ainda, para o uso combinado da estimulação ludomotora com a estimulação cortical, que é um recurso tecnológico que pode ser utilizado para produzir mudanças de padrões cerebrais por condicionamento e/ou interação consciente, providos pelos sistemas visual e/ou auditivo ${ }^{11}$. A estimulação cerebral, neste caso, se dá através do uso de aparelhos eletrônicos que emitem luz e/ou som, no sentido de promover a facilitação de aprendizagem motora ${ }^{12}$.

Os "binaural beats" ou batidas binaurais, emitem dois sons coerentes de frequências quase similares apresentadas com fones estéreos em cada ouvido, sendo neurologicamente transportadas para a formação reticular que regula e controla o nível de estresse, atenção e consciência, fatores preponderantes para uma boa aprendizagem e memorização, pois quando o cérebro modula a frequência em torno de 8 a 12 pulsações por segundo há maiores facilidades de aprendizagem por informações diretas ou subliminares, este é o estado alfa, em que o indivíduo se encontra em estado de relaxamento físico e mental ${ }^{13,14,15}$.

A partir do comentado, o presente estudo teve por objetivo avaliar a importância de um programa ludomotor combinado com a estimulação cortical por vias de batidas "binaural beats", voltado para a melhora do desenvolvimento cinestésicocorporal de crianças com idade entre 5 e 7 anos de idade.

\section{Metodologia}

\section{Tipologia do estudo}

O presente estudo foi desenvolvido por meio de um formato ou desenho experimental e operacionalizado por meio de uma avaliação antes (pré-teste), e outra após (pós-teste) a aplicação de uma intervenção $0^{16}$.

\section{Participantes do estudo}

O grupo participante do estudo foi composto por 150 crianças com idade compreendida entre 5 e 7 anos de idade, estudantes da rede pública e particular de ensino do município de Ariquemes no estado de Rondônia. Como critérios de inclusão no estudo foram selecionados aquelas crianças que não apresentaram disfunções neuromotoras de estrabismo e de déficits auditivos severos e as que não atenderam a esses critérios se utilizou do método de exclusão.

Foram divididas de forma randômica em 3 grupos: Grupo 1 -constituído de 50 crianças que apenas realizaram 0 programa ludomotor; Grupo 2 - constituído de 50 crianças que realizaram o programa de estimulação cortical por via de batidas "binaural beats" em conjunto com o programa ludomotor e Grupo 3 - constituído por 50 crianças que apenas realizaram o programa de estimulação cortical por via de batidas "binaural beats". 


\section{Ética na Pesquisa}

Este estudo foi desenvolvido respeitando as Normas estabelecidas na Resolução 196/96 do Conselho Nacional de Saúde de 10/10/1996 Brasil ${ }^{17}$ com relação à realização de Pesquisa em Seres Humanos, sendo submetido ao Comitê de Ética em Pesquisa do CEULJI/ULBRA e aprovado sob o número de protocolo 043/11.

Todos os participantes do estudo assinaram o termo de consentimento livre e esclarecido (TECLE), no qual constaram os aspectos relativos ao estudo, tais como, objetivo, caráter de voluntariedade da participação e para aderir e/ou sair do estudo, benefícios e possíveis riscos, procedimentos de avaliação, procedimentos de emergência, entre outros.

\section{Procedimentos de avaliacão}

Para a consecução do objetivo do estudo utilizou-se como estratégia de avaliação, o 'Protocolo de avaliação de habilidades motoras de Bruininks - Oseretsky', proposto por Brito et al.,18, que tem como objetivo detectar déficits motores em crianças. Cabe ressaltar que o referido protocolo foi aplicado em dois momentos - um anterior ao desenvolvimento da intervenção e outro posteriormente (Pré X Pós Teste), e que cada criança foi avaliada pelo mesmo avaliador nos dois momentos no período matutino.

O protocolo baseia-se na execução de tarefas relativas a algumas habilidades, que rendem escores que são somados, unificando-se o resultado. As tarefas e seus respectivos escores, que somam no máximo 12 pontos, são:

1 - corrida de Velocidade e Agilidade - consiste numa corrida curta de 13,7 metros envolvendo a captação e o transporte de um objeto ( 0 a 2 pontos).

2 - equilibrio - avalia a habilidade em manter o equilíbrio postural numa posição estática unipodal e num deslocamento dinâmico, ( 0 a 2 pontos).

3 - coordenação Bilateral - avalia a habilidade da criança em coordenar as mãos e os pés em movimento dissociados sequenciais e simultâneos, utilizando ambos os lados do corpo, (0 a 2).

4 - coordenação dos Membros Superiores - avalia as habilidades na recepção bimanual e na coordenação óculomanual de uma bola de tênis dirigida a um alvo ( 0 a 2 pontos).

5 - velocidade de Reação - mede a velocidade de resposta motora a um estímulo visual (régua) em movimento vertical (0 a 2 pontos).

6 - viso-motricidade - avalia a motricidade fina na realização grafo motora de labirintos e de cópias de figuras geométricas ( 0 a 2 pontos).

\section{Procedimentos de intervenção}

Os procedimentos de Intervenção desse estudo foram assim organizados:

a) Programa ludomotor - Consistiu em um programa de exercícios que estimulassem de maneira bio-operacional, a partir de atividades lúdicas, os movimentos básico-fundamentais, os movimentos manipulativos, a consciência corporal (bilateralidade, lateralidade, dominância, equilíbrio) e a imagem corporal, das crianças. 0 referido programa teve a duração de 36 sessões de 35 minutos, realizadas três vezes na semana, num período de três meses.

b) Programa de Estimulação Cortical: teve a mesma duração e periodicidade que o programa ludomotor e foi realizado através de estimulos auditivos por vias de batidas "binaural beats" com a frequência alfa entre 8 e $12 \mathrm{~Hz}$, como normalmente se emprega em estudos desta natureza e que corresponde à frequência que parece estimular as áreas corticais responsáveis pela aprendizagem motora ${ }^{12}$, para o que foi entregue a cada participante um aparelho de MP3 da marca "IPOD" com toda programação adequada e com fone de ouvidos estéreos.

Os alunos executaram o referido programa em uma sala ampla e foram orientados a ficar em silêncio e da maneira mais confortável que desejassem.

Por fim cabe ressaltar que os participantes do grupo 2 que realizaram o programa de estimulação cortical combinado com o programa ludomotor, foram submetidos sempre à estimulação cortical anteriormente ao programa ludomotor.

\section{Tratamento estatístico}

A análise dos dados foi realizada com base na comparação de resultados estatísticos, utilizando-se para isto 0 programa de estatística BIOESTAT 5.0.

Os resultados que foram obtidos foram inicialmente testados, pelo teste de normalidade Shapiro-Wilk, e de acordo com os resultados obtidos neste pôde-se escolher um instrumento paramétrico ou não de análise de Variância para a 
comparação inter-avaliações num sistema intra-grupos através do teste de Wilcoxon e intergrupos através do teste de KRUSKAL-WALLIS, com $\mathrm{p}$ valor $<0.05$.

\section{Resultados}

A partir da tabela 1 pode-se perceber que quando avaliados em relação ao seu desempenho os participantes do grupo 1 (G1), apresentaram inicialmente uma variação de resultados entre 6 e 10 pontos e um desempenho mediano de 7,74 pontos. Já na avaliação após a intervenção a variação de resultados ficou entre 8 e 12 pontos e a mediana dos escores foi de 9,42 pontos.

Tabela 1: Resumo descritivo do protocolo de avaliação de habilidades motoras de Bruininks - Oseretsky
no Grupo 1 pré e pós teste
\begin{tabular}{c|c|c} 
Categoria & G1 Pré & G1 Pós \\
\hline Mínimo & 6.00 & 8.00 \\
\hline Máximo & 10.00 & 12.00 \\
\hline Mediana & 7.74 & 9.42 \\
\hline Variância & 0.80 & 1.22 \\
\hline Desvio Padrão & 0.89 & 1.10 \\
\hline Coeficiente de Variação & $11.62 \%$ & $11.76 \%$ \\
\hline
\end{tabular}

p-valor $=0.02$, Teste de Wilcoxon para amostras pareadas

Nota-se, então, uma evolução dos participantes do grupo 1, comprovada por meio do teste de WILCOXON para duas amostras pareadas, entendendo-se e a referida evolução não foi ao acaso e sim por benefícios da intervenção a que os participantes deste grupo foram submetidos.

Ao observar a tabela 2, percebe-se que os participantes do grupo 2 (G2), que foram submetidos ao programa de estimulação cortical combinado com o programa ludomotor, inicialmente apresentaram um desempenho mediano igual a 7,58 pontos, pois os resultados variaram entre 6 e 10 pontos. Já no instante pós-intervenção a variação de resultados ficou entre 08 e 12 pontos, com mediana de 10,32 pontos, mostrando um efeito positivo da intervenção desenvolvida, resultado também comprovado pelo teste de WILCOXON para duas amostras pareadas.

Tabela 2: Resumo descritivo do protocolo de avaliação de habilidades motoras de Bruininks - Oseretsky do Grupo 2 pré e pós teste

\begin{tabular}{c|c|c}
\hline Categoria & G2 Pré & G2 Pós \\
\hline Mínimo & 6.0000 & 8.0000 \\
\hline Máximo & 10.0000 & 12.0000 \\
\hline Média Aritmética & 7.5800 & 10.3200 \\
\hline Variância & 0.6159 & 1.1608 \\
\hline Desvio Padrão & 0.7848 & 1.0774 \\
\hline Coeficiente de Variação & $10.35 \%$ & $10.44 \%$ \\
\hline
\end{tabular}

p-valor $=0.001$, Teste de Wilcoxon para amostras pareadas

Já os participantes do grupo 3 (G3) obtiveram escore máximo de 10 pontos e o escore e 0 mínimo de 6 pontos e um desempenho mediano igual a 7,72 pontos no pré-teste. Porém, quando avaliados após o período de intervenção, ou seja, após serem submetidos ao programa de estimulação cortical a variação de resultados ficou entre 7 e 12 pontos, com mediana igual a 9,36 pontos.

Ao observar a tabela 3 pode-se perceber uma diferença de 1,64 pontos entre as medianas das duas avaliações, 0 que mostrou significância estatística através do teste de WILCOXON. 
Tabela 3: Resumo descritivo do protocolo de avaliação de habilidades motoras de Bruininks - Oseretsky do Grupo 3 pré e pós teste

\begin{tabular}{c|c|c}
\hline Categoria & G3 Pré & G3 Pós \\
\hline Mínimo & 6.0000 & 7.0000 \\
\hline Máximo & 10.0000 & 12.0000 \\
\hline Média Aritmética & 7.7200 & 9.3600 \\
\hline Variância & 0.9812 & 1.5820 \\
\hline Desvio Padrão & 0.9906 & 1.2578 \\
\hline Coeficiente de Variação & $12.83 \%$ & $13.44 \%$ \\
\hline
\end{tabular}

p-valor $=0.03$, Teste de Wilcoxon para amostras pareadas

Para identificar a amplitude e a direção da diferença entre os grupos, considerando-se os diferentes tipos de intervenção às quais os participantes foram submetidos, foi empregada uma análise de comparações múltiplas através do teste de KRUSKAL-WALLIS que demonstrou significância num sentido de comparação Pré X Pós teste entre o Grupo 2 e Grupo 1. Os resultados também mostraram diferença significativa neste mesmo sentido de comparação entre o Grupo 2 e 0 Grupo 3, o que não aconteceu quando comparado o Grupo 1 com o Grupo 3 (Tabela 4).

\begin{tabular}{c|c|c}
\multicolumn{3}{l}{ Tabela 4: Comparações intergrupos } \\
\hline Comparações & Valor de Z & Valor de $p$ \\
\hline G2 X G1 & 3.7622 & $<0,05$ \\
\hline G2 X G3 & 3.7580 & $<0.05$ \\
\hline G1 X G3 & 0.0058 & Ns \\
\hline
\end{tabular}

Ns: Não apresentou significância.

\section{Discussão}

Acredita-se que os resultados positivos obtidos pelos participantes do grupo 1 (G1) ocorreram provavelmente por uma organização neurofuncional em relação às capacidades cognitivas-motoras, tais como a memória espacial de trabalho e atenção, proporcionando uma melhor integração sensorial entre os mecanismos neurais responsáveis pela junção do perfil cinestésico corporal no desenvolvimento de habilidades motoras fundamentais. Estes resultados são consistentes com pesquisas prévias em contextos de ensino-aprendizagem e desempenho motor como os estudos desenvolvidos pelos autores ${ }^{9,19,20,21}$.

Ao analisar os resultados dos participantes do grupo 2 (G2) que foram submetidos ao programa ludomotor combinado com a estimulação cortical por via auditiva, entendeu-se que este promoveu uma simultaneidade de ativação dos mecanismos neuromotores envolvidos nas tarefas, fazendo desenvolver uma nova ordem para aquisição e maximização do repertório motor dessas crianças, podendo assim, refletir um equilíbrio cerebral mais adequado às funções cognitivas de organização durante tarefa motora ${ }^{10}$.

Os resultados obtidos na mensuração do desenvolvimento motor corroboram com estudos realizados por Calomeni et al. ${ }^{11}$; Tomporowski et al. ${ }^{13}$; Senna et al. ${ }^{14}$; Macedo et al. ${ }^{15}$ e Neto et al. ${ }^{22}$ que também se valeram de estímulos auditivos simultâneos em seus trabalhos e, assim como neste estudo, também obtiveram resultados significativos em suas pesquisas, pelo que propõem ser o cérebro uma estrutura altamente plástica, sendo possível, por meio de estímulo externo auditivo, promover seu balanceamento e aprimoramento das capacidades motoras inerentes a melhora do perfil cinestésico de crianças.

Assim este processo de intervenção combinada, proposto pelo presente estudo pode proporcionar um equilíbrio das funções cognitivas em suas diversas áreas de desempenho motor de suas variadas funções cinestésicas corporais, no que tange à atividade ludomotora através do ato de brincar melhorando a execução das tarefas cotidianas proporcionalmente, 
coerente e adequada, traduzindo-se em harmonia e equilíbrio psicomotor que pode otimizar a aprendizagem e a memorização $0^{23}$.

Portanto pode-se dizer que ao utilizar a frequência entre 8 a $12 \mathrm{HZ}$, faixa que, segundo Cardoso ${ }^{12}$; Ribeiro Junior et al. ${ }^{24}$, está relacionada com a atenção e a aprendizagem, criou-se um cenário cortical propício a este tipo de evento, 0 que se pôde comprovar através da superioridade do grupo 2 sobre os demais grupo deste estudo, confirmando-se o valor ou os efeitos positivos que a estimulação cortical combinado com a intervenção ludomotora, pode trazer ao desenvolvimento cinestésico corporal dessas crianças.

\section{Conclusão}

Pôde-se afirmar que a utilização combinada da estimulação cortical por estímulos auditivos com um programa ludomotor pode ser indicada para facilitar a maturação dos mecanismos relacionados à melhora do desenvolvimento cinestésico corporal de crianças, favorecendo uma melhora de desempenho de tarefas motoras, onde o lúdico combinado com 0 ato motor caracterizado pelo programa ludomotor favorece a maximização da tarefa motora exigida no processo de formação dessas crianças.

Portanto concluiu-se que o treinamento ludomotor combinado com a estimulação cortical aqui proposto é eficaz no desenvolvimento cinestésico corporal dessas crianças melhorando e favorecendo ganhos no seu repertório motor para que se possam realizar tarefas simples e complexas que exijam a integração do seu sistema neuromotor.

Cabe ressaltar que o estudo apresenta algumas limitações como o pouco tempo de intervenção e poucos estudos como referência para que se pudesse obter uma melhor comparação. Assim se recomenda que sejam feitos demais estudos dessa natureza comprovando a importância desse método combinado.

\section{Referências bibliográficas}

1.Lima AP, Cardoso FB, Beresford H, Silva IL. O valor de um programa ludomotor para a melhoria da velocidade de reação de crianças. ConSc Saúd. 2013;12(3):398-404.

2.Awad HZA. Brinque, jogue, cante e encante com a recreação: Conteúdos de aplicação pedagógico teórico/ prático. Várzea Paulista. SP: Fontoura Editora, 2011.

3.Manhães FC, Souza CHM. A estimulação da inteligência corporal cinestésica no desenvolvimento psicomotor na prática da Educação Física escolar. Rev dig. efdeportes.com, Buenos Aires, 2008;13(124):1-1.

4.Carson RG, Smethurst CJ, Oytam Y, Rugy A Postural context alters the stability of bimanual coordination by modulating the crossed excitability of cortico spinal pathways. J Neurophys. 2007;97;2016-2023.

5.Messum P. What if children don't learn to pronounce by imitation? Speak Out. 2008;39:16-21.

6.Raab M, Oliveira RF, Heinen T. How do people perceive and generate options? In Raab M, Hekeren H, Johnson JG. (Eds.) Progress in Brain Research: v. 174. Mind and Motion: The Bidirectional Link between Thought and Action (p. 4959). Amsterdam: Elsevier, 2009.

7.Roerdink M. Anchoring: Moving from theory to therapy. Enschede, the Netherlands: Ipskamp, 2008.

8.Moraes R, Allard F, Patla AE. Validating determinants for alternate foot placement selection algorithm during human locomotion in a cluttered terrain. J Neurophys. 2007;98:1928-1940.

9.Livesey D, Keen J, Rouse J, White F. The relationship between measures of executive function, motor performance and externalising behaviour in 5- and 6-year-old children. Human Moviment Scienc, 2006;25(1):50-64.

10.Williams HG, Pfeiffer KA, Dowda M, Jeter C, Jones S, Pate RR. A Field-Based Testing Protocol for Assessing Gross Motor Skills in Preschool Children: The Children's Activity and Movement in Preschool Study Motor Skills Protocol. Measurement in Physical Education and Exercise Science, 2009;13:151-165.

11.Calomeni MR, Neto NTA, Leal KOF, Silva VF. Estimulação Fótica e Auditiva: Efeitos em Crianças Hiperativas. Rev perspec onl. 2008;5:34-42.

12.Cardoso FB. A utilização do programa de potencialização cerebral para a melhoria no lançamento da bola de boliche para atletas da seleção juvenil colombiana B. Dissertação de Mestrado, Programa Stricto Sensu em Ciência da Motricidade Humana, Universidade Castelo Branco. Rio de Janeiro, 2007. 
13.Tomporowski PD, Davis CL, Miller PH, Naglieri JA. Exercise and Children's Intelligence, Cognition, and Academic Achievement. Education Psychology Review, 2008;20(2):111-131.

14.Senna V, Cardoso F, Silva IL, Beresford H. An evaluation of the effects of cerebral stimulation on motor learning of adolescents practicing judo. Sport Sciences for Health, 2009;5(3):101-104.

15.Macedo LS, Pina IL, Sequeira MEA, Silva IL, Cardoso F, Beresford H. O valor da estimulação cortical voltado para 0 déficit de atenção de alunos com síndrome de Down. Ciênc e Cogniç. 2010;15(3):13-22.

16.Barros AJS, Lehfeld NAS. Fundamentos de metodologia científica: um guia para a iniciação científica. $2^{a}$ ed. São Paulo: Makron Books, 2000.

17.Brasil, Ministério da Saúde. Resolução 196/96 do Conselho Nacional de Saúde/MS Sobre Diretrizes e Normas Regulamentadoras de Pesquisa envolvendo seres humanos. Diário Oficial da União, 10 de outubro, 1996.

18.Brito A. Silva IL, Cardoso F, Beresford H. Avaliação do perfil cinestésico corporal de crianças com Síndrome de Down: um parâmetro para se atender à proposta das Diretrizes Curriculares Nacionais para a educação especial. Ensaio: aval polític públic Educac. Rio de Janeiro, 2009;17(63):341-354.

19.Piek JP, Dawson L, Smith LM, Gasson N. The role of early fine and gross motor development on later motor and cognitive ability. Human Moviment Scienc. 2008;27(5):668-681.

20.Lopes V, Rodrigues L, Maia J, Malina R. Motor coordination as predictor of physical activity in childhood. Scand J Med Sci Sports. 2011;21(5):663-9.

21.Lopes LO, Lopes VP, Santos R, Pereira BO. Associações entre atividade física, habilidades e coordenação motora em crianças portuguesas. Rev. Bras Cineantr Desemp Hum. 2011;13(1):15-21.

22.Neto NTA, Morales AP, Calomeni MR, Viana MAS, Silva VF. Estimulação cortical: efeitos agudos sobre variáveis bioperacionais em jogadores armadores de basquetebol. Rev dig. efdeportes.com, Buenos Aires, 2010;15(150):1-1.

23.Niederer I, Kriemler S, Gut J, Hartmann T, Schindler C, Barral J, Puder JJ. Relationship of aerobic fitness and motor skills with memory and attention in preschoolers (Ballabeina): A cross-sectional and longitudinal study. BMC Pediatrics, 2011;11(34).

24.Ribeiro Júnior SMS, Azevedo MMA, Jorge FS, Morales AP, Silva VF. Efeito da estimulação cortical na variável tempo de reação e performance hábil-motriz-cognitiva em jovens atletas de futebol. Motriz: Rev. Educ Fís. São Paulo, 2010;16(4):869-879.

\section{Alisson Padilha de Lima}

Endereço para correspondência - Avenida Silvio Romero, 230, apto 108. Bairro São José, CEP: 99051-150, Passo Fundo, RS, Brasil.

E-mail: professor.alissonpadilha@gmail.com

Lattes: http://lattes.cnpq.br/8408892033315305

Fabrício Bruno Cardoso - fabriciobrunocardoso@gmail.com

Enviado em 01 de abril de 2014

Publicado em 17 de outubro de 2014 
